\title{
COMPARING THE PERFORMANCE OF POINT CLOUD REGISTRATION METHODS FOR LANDSLIDE MONITORING USING MOBILE LASER SCANNING DATA
}

\author{
Ahmad Fuad N., Yusoff A.R, Ismail Z and Majid Z \\ Geospatial Imaging and Information Research Group, Faculty of Built Environment and Surveying, Universiti Teknologi Malaysia - \\ (nursyahiraaf, ahmadrazali89)@gmail.com, (zamriismail, zulkeplimajid)@utm.my
}

KEY WORDS: point clouds, registration methods, landslide monitoring, mobile laser scanning, deviation maps

\begin{abstract}
:
The aim of the research is to evaluate the performance of the point cloud registration methods using mobile laser scanning data. The point cloud registration methods involved in this research are match bounding-box centres and iterative closest point (ICP). The research began with the two epoch's mobile laser scanning survey using a Phoenix AL-3-32 system. At the same time, the stereo images of the study area were acquired using UAV Photogrammetric method. Both two epoch point cloud datasets were gone through the pre and post-processing stages to produce the cleaned and geo-referenced point clouds data. The data were then gone through the two registration methods and four Cloud-to-Cloud $(\mathrm{C} 2 \mathrm{C})$ distance methods. The 3D surface deviation results obtained from mobile laser scanning data was compared with the 3D surface deviation results from UAV data that undergoes the same registration and $\mathrm{C} 2 \mathrm{C}$ distance computation methods. The study area involved in the research is an active landslide area that was located at Kulim Hi-Tech residential area in Kedah state, Malaysia. The study area exposed to the movement of the land which caused cracked to the buildings and drainages. The findings show that the ICP registration becomes the most suitable method to register point clouds dataset that was acquired using mobile laser scanning system. Among the four C2C distance computation methods that was involved in the testing, the least square plane method was the best method to calculate the distance between two sets of point clouds datasets which in turn gave the best results in the process of detecting the movement of the land in the study area.
\end{abstract}

\section{INTRODUCTION}

Landslide is one of the most common disasters in Malaysia. Factors that lead to this incident are due to natural and human activities. Therefore, it is important to monitor landslides to be overcome quickly and systematically. One of the latest geospatial mapping technology is three-dimensional laser scanning. The technology provides fast, rapid and 3D data with survey grade accuracy. Due to the rapid changes of the landslide surface, 3D laser scanning technology has become the most appropriate solution for data collection phase as the technology can perform the scanning task between epochs in short period of time. Therefore, the research was carried out using mobile laser scanning technology, as geospatial data collection method to acquire $3 \mathrm{D}$ surface data of the selected landslide area.

The aim of the research was to evaluate the performance of the point cloud registration methods to generate three-dimensional (3D) deviation analysis for landslide monitoring using mobile laser scanning data. The research involves with two registration methods which are matching bounding-box centres and fine registration (iterative closest point). These two methods were currently embedded in open-source point cloud processing software known as CloudCompare. The research also involves with the evaluation of cloud-to-cloud distance methods which are nearest neighbour, and the three local modelling methods which are least square plane, $2.5 \mathrm{D}$ triangulation and quadric.

\section{LITEARATURE REVIEW}

\subsection{Mobile laser scanning}

Light detection and Ranging (LiDAR) is a new technology for collecting three-dimensional surface data of an object. Nowadays, the LiDAR technology can be categories in three main categories which are airborne-based LiDAR, terrestrialbased LiDAR and mobile-based LiDAR. The mobile-based LiDAR or popularly known as Mobile Laser Scanning (MLS) becomes the latest LiDAR system where the three-dimensional point cloud of the object was collected from the moving laser scanner setup on the vehicle. Mobile laser scanning (MLS) starts with the stop-and-go scanning mode to collect the point cloud data. Nowadays, the innovation in the MLS system makes the system running of the on-the-fly mode. Not only that, the current MLS system can be carried by human for data collection at the un-access area. Figure 1 show the concept applied in MLS surveying.

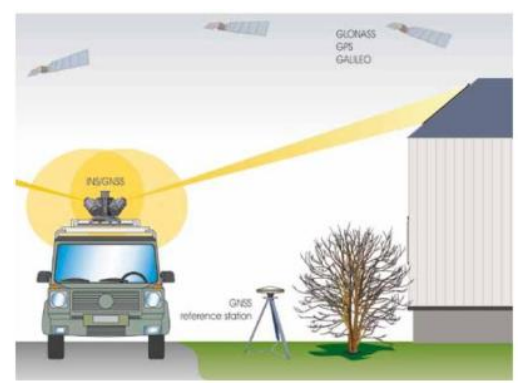

Figure 1. The concept of mobile laser scanning survey (Wang $\mathrm{H}$ et. al (2012)) 


\subsection{Methods for point cloud registration}

There are few methods that have been developed for the point cloud registration. The developed registration methods were embedded in either commercial or open source software. One of the open source software that can be used for point cloud registration is CloudCompare software. The most common registration methods offered by the cloudcompare software are match bounding-box centres and iterative closest point (ICP). The detail of each method is discussed below.

\subsubsection{Matching Bounding-Box Centres Registration Method}

The Match Bounding-Box Centres (will be known as MBBC) registration method is the simplest point cloud registration method that translate all selected entities (point cloud datasets) so that their bounding-box centres will be mapped at the same place. One of the selected entities (point cloud data) will be used as reference data and the second entity will be mapped to the centre of the reference data. The $4 \times 4$ transformation matrix that corresponded to the applied translation will be computed. Figure 2 shows the registration process using match boundingbox centres method, while Figure 3 shows the $4 \times 4$ transformation matrix for the applied translation process.

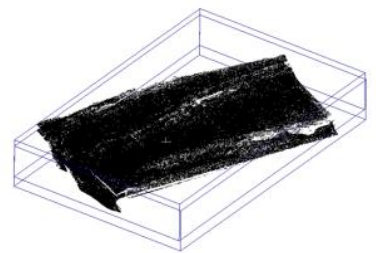

(a)

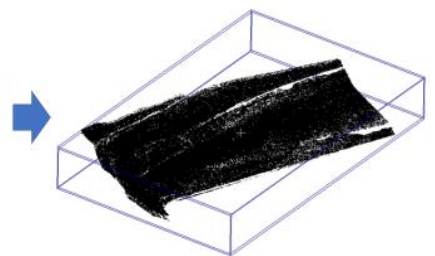

(b)
Figure 2. Point clouds registration using MBBC method - (a) before registration process, (b) after registration process (perspective view)

\begin{tabular}{l}
\hline Match Bounding-Box Centers - Computed Transformation Matrix \\
1.0000000000000 .0000000000000 .0000000000000 .281250000000 \\
$0.0000000000001 .0000000000000 .000000000000-0.023437500000$ \\
$0.0000000000000 .0000000000001 .000000000000-2.659999847412$ \\
0.0000000000000 .0000000000000 .0000000000001 .000000000000 \\
\hline
\end{tabular}

Figure 3. The $4 \times 4$ transformation matrix for the applied translation process for $\mathrm{MBBC}$ registration method

\subsubsection{Iterative Closest Point (ICP) Registration Method}

Iterative Closest Point (will be known as ICP) is one of the most popular method for the registration of deformed and undeformed point clouds data. According to Jafari (2016), the overall aim of the ICP algorithm is to estimate a rigid transformation between $p_{i} \in P$, a point from the reference $3 \mathrm{D}$ point cloud, and $q_{i} \in Q$, a point from the target point cloud. The ICP method implements nearest neighbours and Euclidean distance calculation and estimates the closest point between the $p_{i}$ and $q_{i}$ as correspondence points. Figure 4 shows the correspondence estimation between undeformed reference point cloud data $\mathrm{P}$ and deformed point cloud data $\mathrm{Q}$.

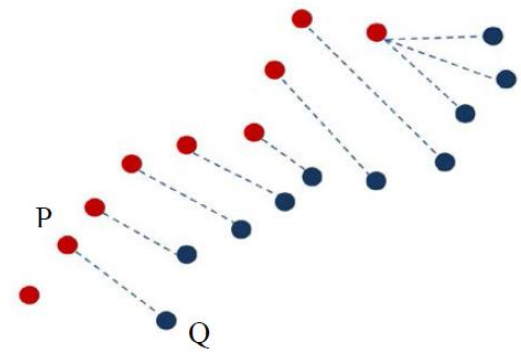

Figure 4. Correspondence estimation between undeformed reference point cloud data $\mathrm{P}$ and deformed point cloud data $\mathrm{Q}$

(Jafari, 2016)

In order to calculate the rotation $\mathrm{R}$ and translation $\mathrm{t}$ between $p_{i}$ and $q_{i}$, the ICP method uses an error function to minimize the sum of the square distances. Equation 1 shows the error function formula use in ICP method.

$$
E(R, t)=\min _{R, t} \sum_{i}\left\|p_{i}-\left(R q_{i}+t\right)\right\|^{2}
$$

where

$p_{i} \in P=$ a point from $3 \mathrm{D}$ reference point cloud $q_{i} \in Q=$ a point from target point cloud

Once the point clouds datasets are spatially registered and scaled, the deformation deviation analysis can be performed using cloud to cloud distance computation method.

\subsection{Cloud-to-Cloud Distance Computation Method}

One of the most common cloud distance computation method is Cloud-to-Cloud method (will be known as $\mathrm{C} 2 \mathrm{C}$ method). C2C method is the computation of distances between two clouds or between a point cloud and a mesh. The purpose of $\mathrm{C} 2 \mathrm{C}$ method in this study is to determine the distance difference between two epochs of mobile laser scanning data. The distance differences were referring to the movement of land slip occurred at the study area. Figure 5 shows the basic concept of $\mathrm{C} 2 \mathrm{C}$ computation method.

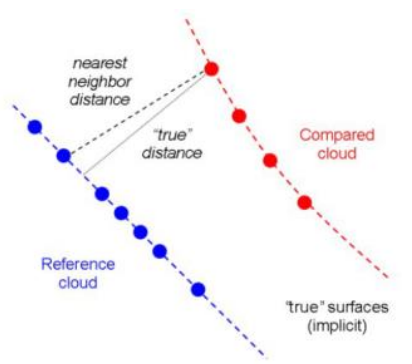

Figure 5. The basic concept of $\mathrm{C} 2 \mathrm{C}$ distance computation method

The basic $\mathrm{C} 2 \mathrm{C}$ distance computation method calculate nearest neighbor distance between the reference cloud and the compared cloud datasets. The principle of nearest neighbor distance is used to compute the distances between the two points where for each point in the compared cloud, the nearest point in the reference cloud is searched and their Euclidean distance is computed. In order to get better approximation of the true distance to the reference surface, the local surface model was introduce. Figure 6 shows the concept used in local model $\mathrm{C} 2 \mathrm{C}$ distance computation. 


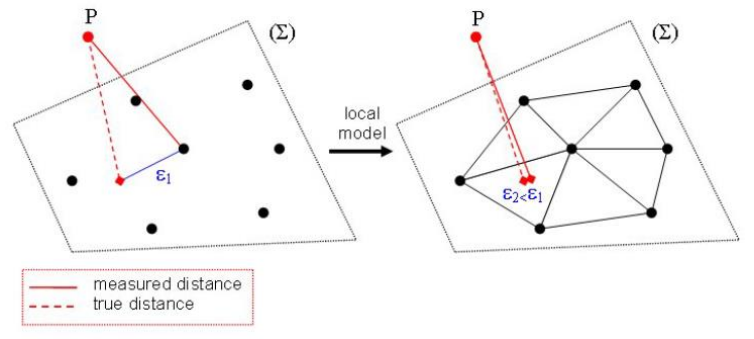

Figure 6. The concept of local surface model C2C distance computation method

Local surface model methods work by locally model the surface of the reference cloud by fitting a mathematical primitive on the nearest point and several of its neighbours. This process was carried out when the nearest point in the reference cloud is determined. CloudCompare software offers three local surface model methods which are least square palne, 2D1/2 triangulation and quadric. The effectiveness of the local surface model is statstically more or less dependent on the cloud sampling and on how appropriate the local surface approximation is (Shen et. Al, 2017).

According to Jafari (2016), the C2C distance computation algorithm implements the Hausdorff distance that calculate the distances between the correspondence points. The Hausdorff distance from set $\mathrm{A}$ to set $\mathrm{B}$ is a maximum function defines as Equation 2 below:

$$
H(A, B)=\max _{a \in A}\left\{\min _{b \in B}\{d(a, b)\}\right\}
$$

where

$$
\begin{array}{ll}
a & =\text { points of set } A \\
b & =\text { points of set } B \\
d(a, b) & =\text { any metric between these points. }
\end{array}
$$

\subsection{Previous research involved with 3D surface deviation analysis}

Three-Dimensional surface deviation analysis between clouds can be implemented by using various methods of registration and surface change detection either embedded in the open source or commercial software. The related study about these was mentioned in Barnhart and Crosby (2013) about the methods of Cloud to Mesh (C2M) and Multiscale Model to Model Cloud Comparison (M3C2) were used to analyse surface change detection. Successfully proved that the M3C2 method provides better results in displacement measurement compared to $\mathrm{C} 2 \mathrm{M}$ method where $\mathrm{M} 3 \mathrm{C} 2$ manage to calculate the true horizontal displacements of Terrestrial Laser Scanning (TLS) data while $\mathrm{C} 2 \mathrm{M}$ could not but manage to use the threshold of change detection. The effectiveness of $\mathrm{M} 3 \mathrm{C} 2$ method also supported from Moghaddame-Jafari (2017) where the algorithm of M3C2 gave the sub-millimetre accuracy $(0.4 \mathrm{~mm})$ in vertical deflection measurement but the importance of correct registration and alignment of clouds need to be considered due to the sensitivity of registration errors.

Haugen (2016) study about the comparison analysis between qualitative and quantitative in the displacement measurements of 3D LiDAR landslide data. Two registration methods of quantitative analysis were carried out by using Iterative Closest Point (ICP) and 3D Particle Image Velocimetry (3DPIV) to detect the translational slow-moving landslide. 3DPIV registration method shown more accurate and precise result than
ICP method due to the less effect from vegetation growth and processing time. The complex of vegetation growth becomes problematic to the ICP windowed but it can be minimized by increasing the interval of landslide interest data collection.

Oniga et al., (2016) also stated the importance to do the registration part as accurate as possible before performing the surface deviation analysis between clouds. Tie point-based registration method embedded in CloudCompare software is used to analyse the TLS data then carried out the accuracy evaluation by compared with the five pairs of point chosen and measured manually. The registration parameters between those two clouds were then estimated by using 3D conformal transformation and least squares methods which proved the methods can be used for 3D surface deviation analysis. From these several previous studies, different methods of registration and different surface change detection displacement were evaluated in different ways and showed various of results. Some of the methods might be suitable for certain study area and some might be less suitability.

Manousakis et al (2016) carried out a research on the comparison of UAV-enabled photogrammetry-based 3D point clouds and interpolated DSM of sloping terrain for rockfall hazard analysis. The comparison procedure was utilized using CloudCompare software. The results show that the $3 \mathrm{D}$ surface deviation method is the most suitable method to detect the changes of the area due to the rockfall phenomenon.

Hence, this research will focus more on analysing the 3D surface deviation of two epoch's MLS data using two different registration methods and four $\mathrm{C} 2 \mathrm{C}$ distance methods.

\section{METHODOLOGY}

The methodology of the research comprises of five phases. The phases are area data collection, processing of point cloud raw data, point cloud registration, surface deviation analysis and analysis of findings. Below are the complete explanations of each phase.

\subsection{Phase I: Mobile Laser Scanning Data Acquisition}

As mentioned earlier elsewhere in the paper, the acquisition of 3D point cloud data of the landslide surface was carried out using mobile laser scanning (MLS) system. The MLS system known as Phoenix AL3-32 was one of the latest LiDAR system that was developed to acquire point cloud data with survey grade accuracy. The Phoenix AL3-32 system is able to be operated in two MLS data acquisition modes which are vehiclebased mode and human-based mode. In this research, both vehicle-based and human-based mode was used to complement each other to acquire complete surface of the study area. Figure 7 shows the MLS data acquisition modes that were implemented in the research.
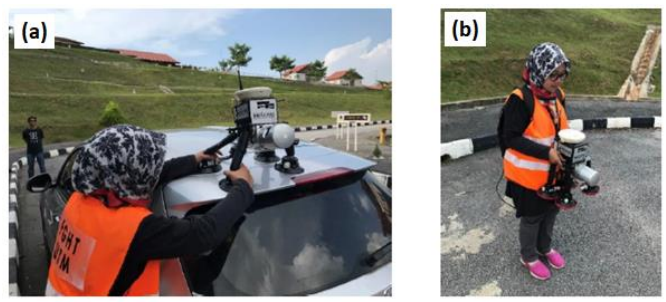

Figure 7. Mobile laser scanning (a) vehicle-based mode and (b) human-based mode 
The acquired MLS data consists of three data. The first data was acquired using vehicle-based mode. Due to the multisloped form of the landslide area, the vehicle-based mode was not being able to scan part of the area. The other two data were acquired using human-based mode. Figure 8 shows the three MLS data that was successfully acquired to fully cover the landslide area.
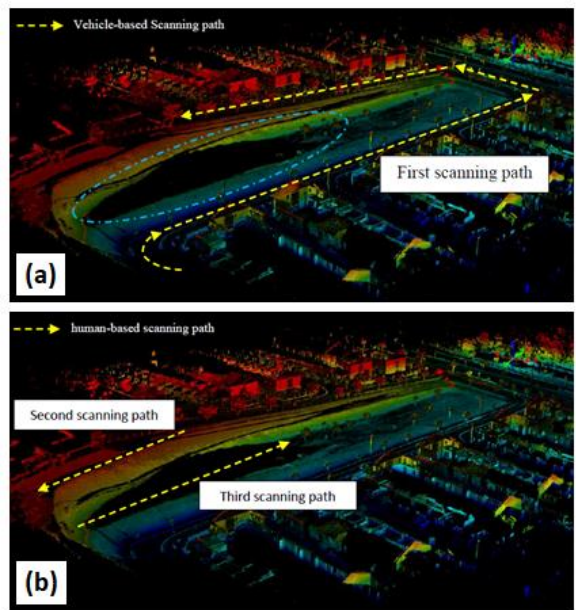

Figure 8. Mobile laser scanning data of the study area - (a) point cloud data acquired using vehicle-based mode; (b) point cloud data acquired using human-based mode

\subsection{Phase II: Processing of point cloud raw data}

The second stage involves in the research is the processing of point cloud raw data that was acquired using mobile laser scanning technology (as mentioned in section 3.1). The processing tasks involves with the cleaning, filtering and merging of three-dimensional point cloud data using GIS spatial analysis methods.

The cleaning process was then applied to the overall scanning data. The purpose of the cleaning process is to delete the unused point cloud data that belong to the man-made objects such as houses, trees and others. The data cleaning process was carried out manually. Figure 9 shows the point cloud data that has been cleaned from the overall scanning data.

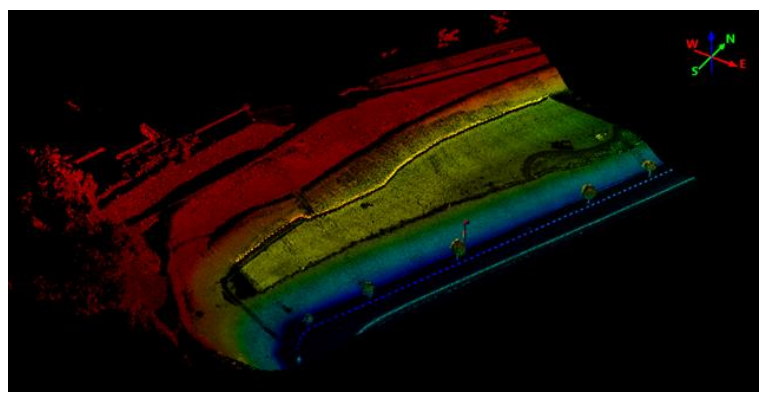

Figure 9. Mobile laser scanning data after cleaning process

The point cloud data (as shown in Figure 9) was then filtered using Adaptive TIN method. The purpose of the filtering method is to separate the ground point cloud data from the nonground data. The final output is the ground point cloud data of the study area. The filtering process was carried out using TerraScan software. The Adaptive TIN filtering method requires special parameters to perform the filtering process. Table 1 shows the parameters and the selected values that have been used in filtering the point cloud data.

\begin{tabular}{|l|c|}
\hline \multicolumn{1}{|c|}{ Parameter } & Value \\
\hline Max. building size & $40.0 \mathrm{~m}$ \\
\hline Terrain angle & $50^{\circ}$ \\
\hline Iteration angle & $3.5^{\circ}$ to plane \\
\hline Iteration distance & $0.5 \mathrm{~m}$ to plane \\
\hline Reduce iteration angle when & $1.0 \mathrm{~m}$ \\
\hline
\end{tabular}

Table 1. Selected parameters for the filtering process using Adaptive TIN method

The selection and determination of values for each parameter are referring to the actual situation of the study area. The results of the filtering process are shown in Figure 10.

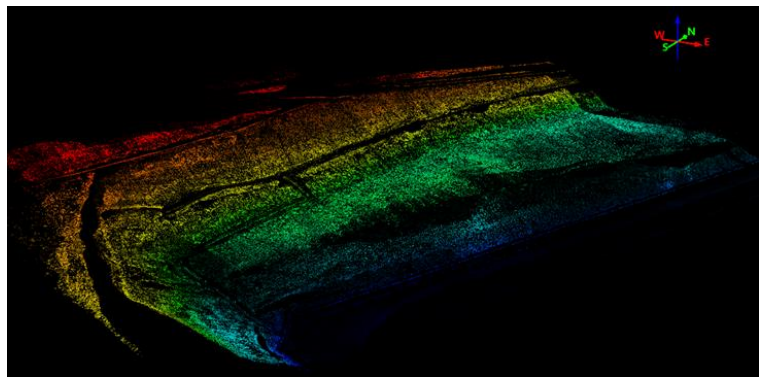

Figure 10. Filtered mobile laser scanning data

The final step in the processing of mobile laser scanning data is a merging process. The purpose of the merging process is to accurately merge the three sets of point cloud data that has been acquired and filtered. The merging process was carried out using a merging algorithm that was provided in the geoprocessing tools embedded in ArcGIS software. Figure 11 shows the mobile laser scanning data before merging process. While Figure 12 shows the final result of the merging process.

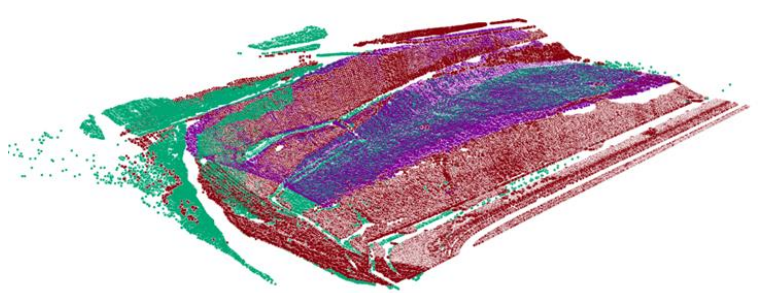

Figure 11. Mobile laser scanning data before merging process

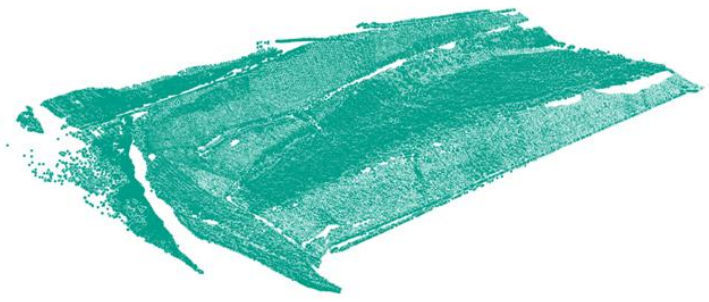

Figure 12. Result for the merging process

Table 2 summarized the chronology of the mobile laser scanning data processing tasks in the aspect of the density of 3D points. The two epoch's mobile laser scanning data was processed separately. 


\begin{tabular}{|l|c|c|}
\hline \multicolumn{1}{|c|}{ Chronology } & Epoch 1 & Epoch 2 \\
\hline All points (RAW data) & 151314709 & 179634130 \\
\hline After Crop & 99286106 & 116976329 \\
\hline After Filter & 382029 & 390197 \\
\hline After Merge & 325185 & 357745 \\
\hline
\end{tabular}

Table 2. The chronology of the mobile laser scanning data processing tasks

Table 2 shows that the density of the point cloud data started to largely reduced when the data was filtered. The situation is happening caused by the removal of non-ground points from the original dataset. As clearly shown in Table 2 that the merging process was also reduce the density of the filtered data caused by the removal of the redundant points in each dataset. The final mobile laser scanning data is the $3 \mathrm{D}$ point clouds data that only belong to the terrain features of the study area.

For the purpose of the surface deviation analysis process, the merged mobile laser scanning data (as shown in Figure 12) was gone through the second stage of data cleaning process. In this process, the unnecessary point cloud data that not belong to the landslide surface was manually deleted. Figure 13 shows both epoch 1 and epoch 2 mobile laser scanning data after the second stage of data cleaning process.
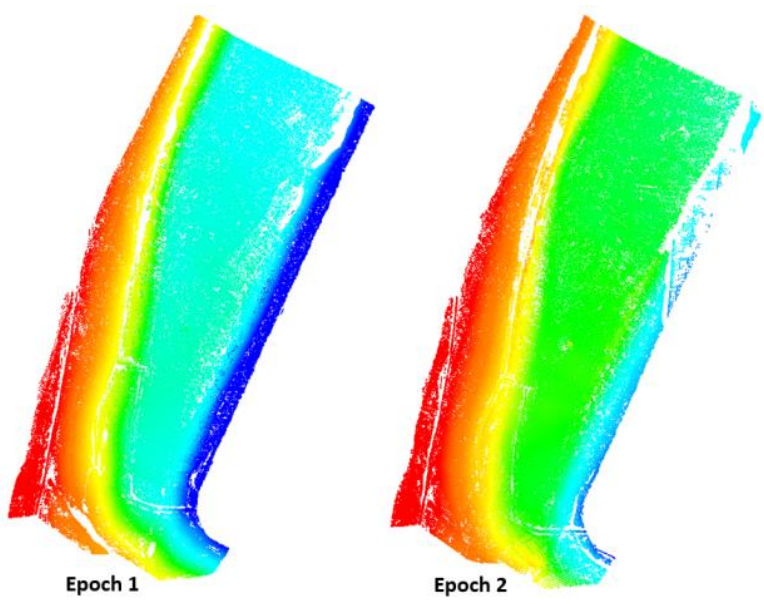

Figure 13. Both epoch 1 and epoch 2 mobile laser scanning data after the second stage of data cleaning process

\begin{tabular}{|c|c|c|}
\hline Epoch & $\begin{array}{c}\text { Number of point } \\
\text { clouds }\end{array}$ & Precision (mm) \\
\hline \multirow{2}{*}{1} & \multirow{2}{*}{299,017} & $(\mathrm{X}) 0.001900$ \\
\cline { 3 - 3 } & & $(\mathrm{Y}) 0.003800$ \\
\cline { 3 - 3 } & \multirow{3}{*}{325,328} & $(\mathrm{Z}) 0.000500$ \\
\cline { 3 - 3 } & & $(\mathrm{Y}) 0.001900$ \\
\cline { 3 - 3 } & & $(\mathrm{Z}) 0.00005000$ \\
\hline
\end{tabular}

Table 3. Metadata for epoch 1 and epoch 2 mobile laser scanning data after the second stage of data cleaning process

\subsection{Phase III: Point Cloud Registration}

Two methods were used to perform the point clouds registration process. The two methods are:

- Match Bounding-Box Centres (MBBC); and

\section{- $\quad$ Iterative Closest Point (ICP)}

In this research, the two epochs MLS datasets was first registered using MBBC registration method. Figure 14 shows the MBBC registration process. The details information of the MBBC registration method is discussed in section 2.2.1.

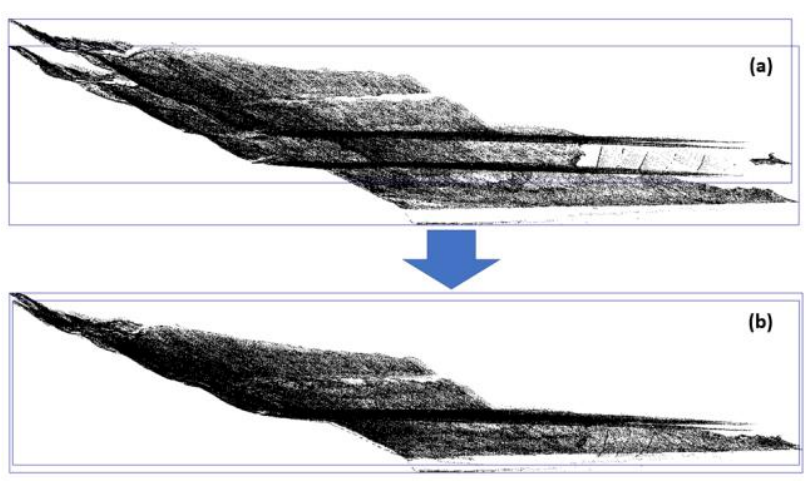

Figure 14. Point clouds registration using MBBC method - (a) before the registration process, (b) after the registration process

The second method used for the registration of the two epochs MLS datasets is the ICP registration method. The details information on the ICP is discuss in section 2.2.2. CloudCompare software provides a capability to perform the ICP registration process automatically. User needs to set the value for the number of iterations and the point sampling unit before executing the ICP registration process. Figure 15 shows the ICP registration menu offers by the CloudCompare software.

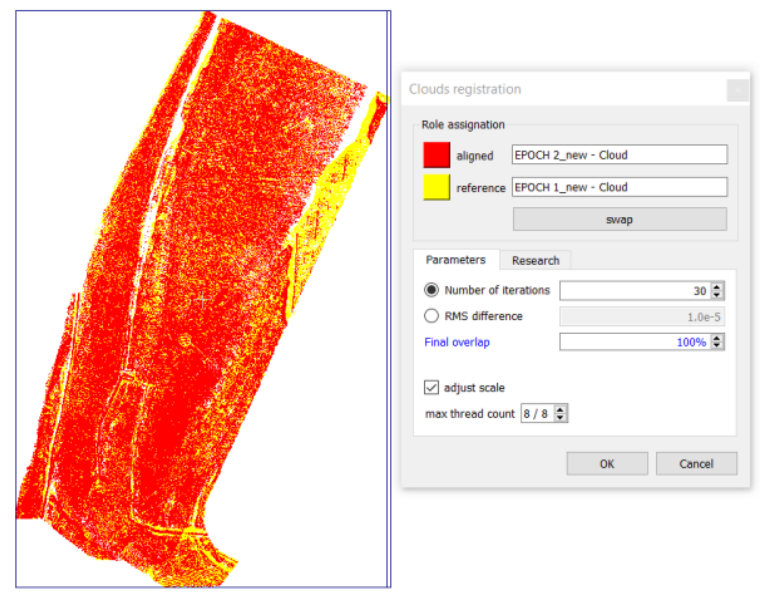

Figure 15. ICP registration process menu in CloudCompare software

The calculated results for the ICP registration method was than appeared in the dialogue box as shown in Figure 16 below. The calculated results show the RMS value, the number of clouds points involved in the calculation, the transformation matrix, the scale and percentages of the overlap. 


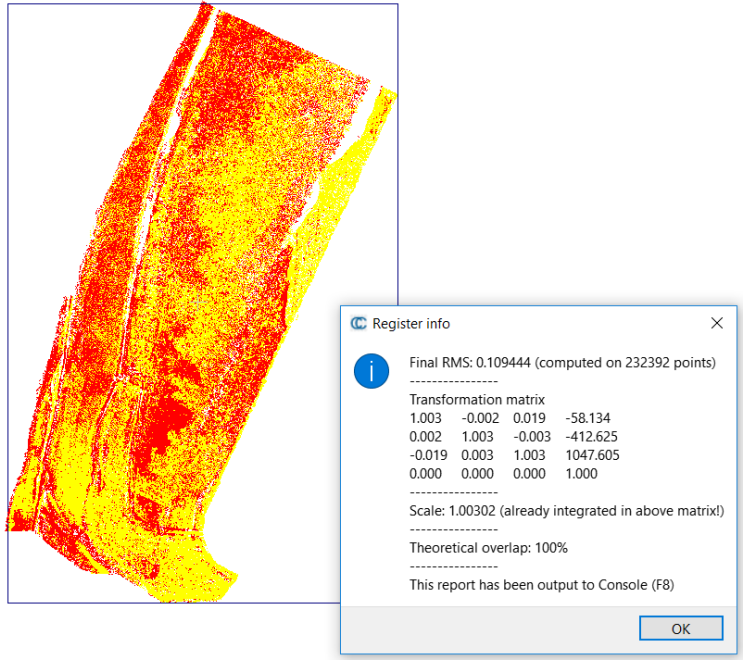

Figure 16. ICP registration result

Both MBBC and ICP methods allowed the user to set the reference dan the compared datasets to be used in the registration process. In CloudCompare software, both methods were setup to process the data automatically. The time for the processing task depends on the size of the point clouds datasets. Hugh size of datasets will take longer time to process as compared to the small size of datasets.

\subsection{Phase IV: 3D Surface Deviation Analysis}

In this research, the 3D surface deviation analysis is carried out using the $\mathrm{C} 2 \mathrm{C}$ distance computation method via CloudCompare software. The first step involve is the determination and the selection of the reference and the compared datasets. The reference dataset is the epoch 1 data and the compared dataset is the epoch 2 data (as shown earlier in Figure 15). Figure 17 shows the selection of datasets to be used in the $\mathrm{C} 2 \mathrm{C}$ distance computation process.

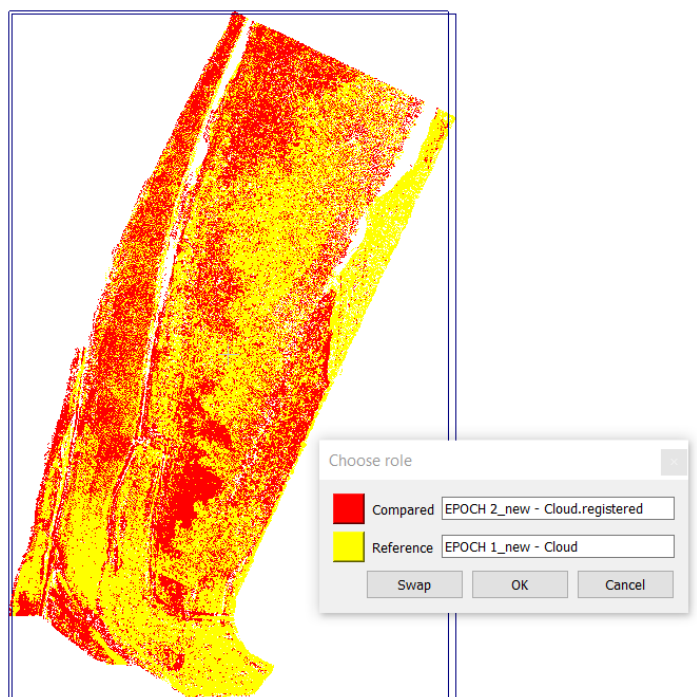

Figure 17. The selection of datasets to be used in the $\mathrm{C} 2 \mathrm{C}$ distance computation process

The CloudCompare software will firstly calculates the approximate results for the distance computation between the selected datasets. The nearest neighbor $\mathrm{C} 2 \mathrm{C}$ distance computation can be carried out using the default settings by choosing the Compute button. The default settings will automatically calculate the suitable Octree level value that is suitable to be used in the calculation process. The 3D surface deviation result is shown and stored in epoch 2 datasets where the user can easily visualize the result by activated the data in the layer panel. Figure 18 shows the $\mathrm{C} 2 \mathrm{C}$ distance computation menu provide by the CloudCompare software.

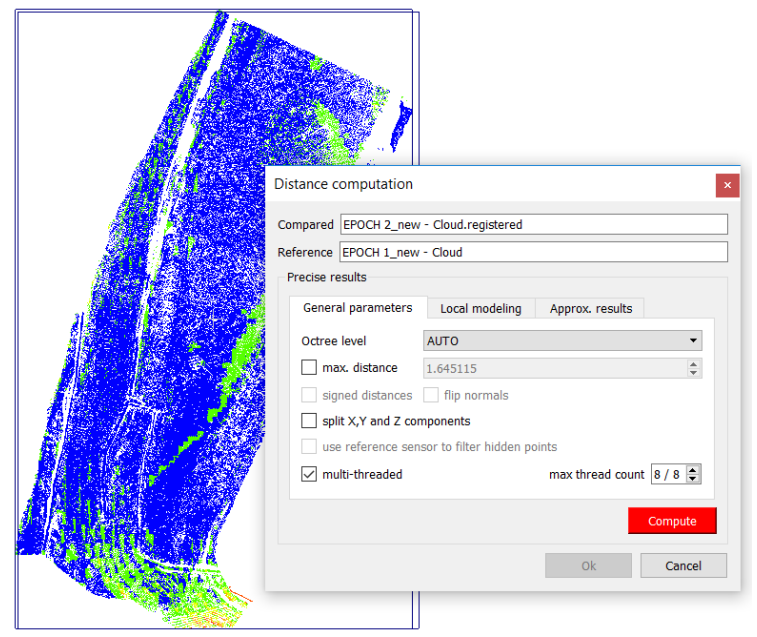

Figure 18. C2C distance computation menu provide by the CloudCompare software

As mentioned earlier elsewhere in the paper, the CloudCompare software offers C2C distance computation through local surface model methods. User can choose and select the local surface model methods from the local modelling menu. Figure 19 shows the menu for selecting the local surface model methods in CloudCompare software.

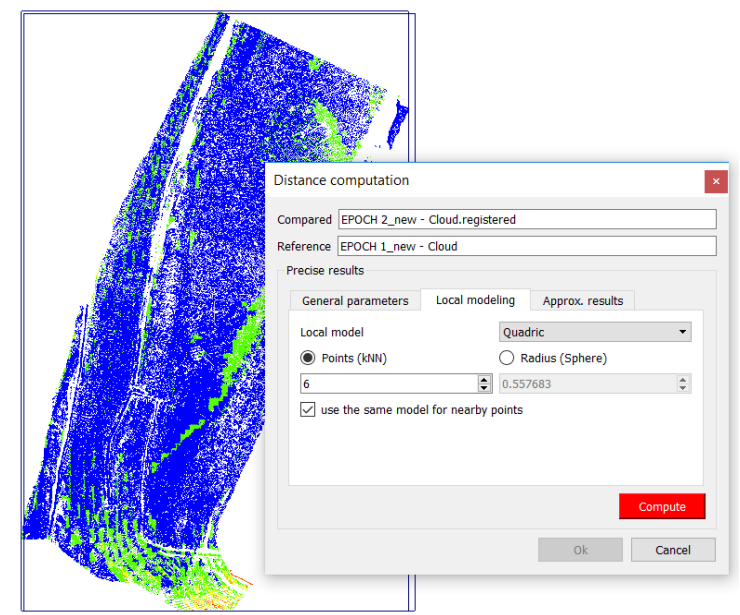

Figure 19. C2C distance computation - selection of local surface model

The local surface model $\mathrm{C} 2 \mathrm{C}$ distance computation was calculated automatically by using the compute button that appeared in the software. Again, the 3D surface deviation result is shown and stored in epoch 2 datasets where the user can easily visualize the result by activated the data in the layer panel. 
The final output from the 3D surface deviation analysis is the deviation analysis result. The result can be used to detect the changes due to the movement that occurs in the compared dataset as a difference to the reference dataset. For better understanding of the result, CloudCompare software provides a colour scale that shows the value of the $\mathrm{C} 2 \mathrm{C}$ distance computation (as shown in Figure 20).

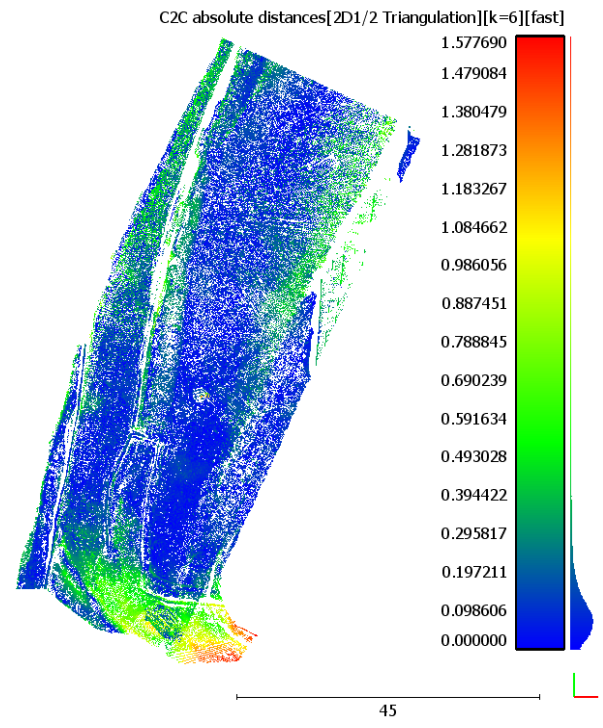

Figure 20. 3D surface deviation analysis result - the C2C distance computation values in colour scale mode

The $\mathrm{C} 2 \mathrm{C}$ distance computation process using CloudCompare software was exposed to many errors. One of the most common errors is the problem on the results due to the un-necessary point clouds data that are not belong to the computed surfaces. For MLS datasets, the used of the most suitable filtering method is highly needed in order to produce the point clouds datasets that only belong to the ground surface.

\subsection{Phase V: Analysis of Results}

In this research, the study area was also mapped using UAVPhotogrammetric method. The series of stereo images was acquired using Phantom 4 DJI drone system at the similar epochs. The images were than processed using structure from motion (SfM) method to produce the point cloud data of the study area. Figure 21 show the Phantom 4 DJI drone system used in the research.

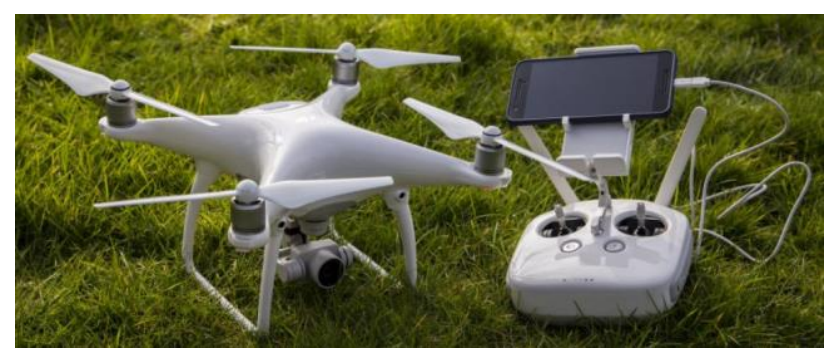

Figure 21. UAV-Photogrammetric Drone System - Phantom 4 DJI System

The ICP registration method were than used to register the two epochs point cloud data and the process was end up with the
$\mathrm{C} 2 \mathrm{C}$ distance computation to show the movement area on the landslide surface. Figure 22 shows the UAV-photogrammetric point cloud data and the $\mathrm{C} 2 \mathrm{C}$ distance computation output.

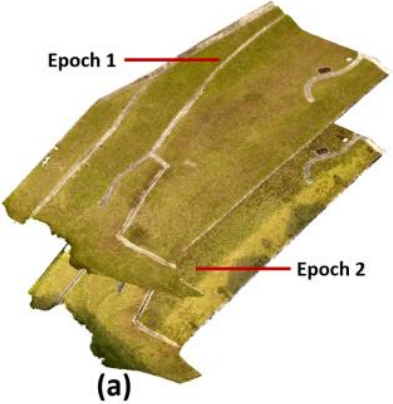

(a)

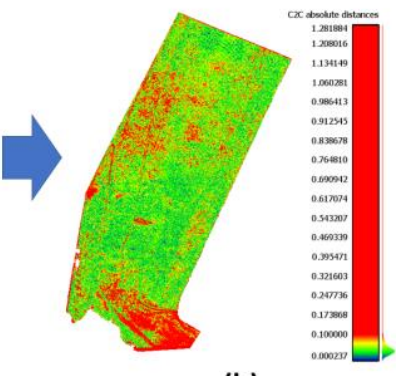

(b)
Figure 22. Analysis using UAV-Photogrammetric data - (a) the UAV-photogrammetric point clouds data and (b) the $\mathrm{C} 2 \mathrm{C}$ distance computation output

The 3D surface deviation analysis result that was produced from the UAV-Photogrammetric data (as shown in Figure 22 (b) were than compared with the 3D surface deviation analysis results that was produced from the MLS datasets.

The analysis also involves with the analysis of $\mathrm{C} 2 \mathrm{C}$ distance computation methods in order to find the most suitable point clouds registration method for MLS datasets. The analysis involves with four $\mathrm{C} 2 \mathrm{C}$ distance computation methods which are nearest neighbor, least square plane, 2D1/2 triangulation and quadric. The data was processed five times and the average of the standard deviation of the $\mathrm{C} 2 \mathrm{C}$ distance computation method was calculated. The complete analysis of results was shows in section 4.3.

\section{RESULTS, ANALYSIS AND DISCUSSION}

The results of the research can be divided into two parts which are (a) point cloud registration results and (b) 3D surface deviation analysis results. As mentioned elsewhere in the paper, the research only involves with common point cloud registration methods which are match bounding-box centres and iterative closest point. Also mentioned that the cloud to cloud distance computation method is the only method used for analysing the surface deviation between the two epochs of point cloud datasets. The details of the results are discussed below.

\subsection{Point cloud registration results}

\begin{tabular}{|c|c|c|c|}
\hline \multicolumn{4}{|c|}{$\begin{array}{c}\text { Parameters for Match Bounding-Box Centers } \\
\text { Registration Method }\end{array}$} \\
\hline Number of point clouds for Epoch 1 & 299,017 points \\
\hline Number of point clouds for Epoch 2 & 325,328 points \\
\hline \multicolumn{3}{|c|}{ Octree level (auto) } & \multicolumn{2}{|c|}{8} \\
\hline \multicolumn{4}{|c|}{$\begin{array}{l}\text { Calculated Parameters for Match Bounding-Box } \\
\text { Centers Registration Method }\end{array}$} \\
\hline $\begin{array}{c}\text { Box dimension } \\
\text { for Epoch 1 }\end{array}$ & X: 60.3984 & Y: 92.8984 & $\mathrm{Z}: 13.7$ \\
\hline $\begin{array}{c}\text { Box dimension } \\
\text { for Epoch 2 }\end{array}$ & X: 59.8984 & Y: 92.9531 & Z: 12.54 \\
\hline $\begin{array}{c}\text { Box center } \\
\text { coordinates }\end{array}$ & X: 73941.2 & Y: 96521.1 & Z: 31.72 \\
\hline
\end{tabular}

Table 4. Summary of the parameters involve in MBBC registration method 
The final results for the MBBC registration method is the $4 \mathrm{x} 4$ transformation matrix as shown in Figure 23. While Figure 24 shows the registered point cloud data.

\subsection{0 .0000000000000 .0000000000000 .281250000000 $0.0000000000001 .0000000000000 .000000000000-0.023437500000$ $0.0000000000000 .0000000000001 .000000000000-2.659999847412$ 0.0000000000000 .0000000000000 .0000000000001 .000000000000}

Figure 23. The $4 \times 4$ transformation matrix as a result from the match bounding-box centers registration method

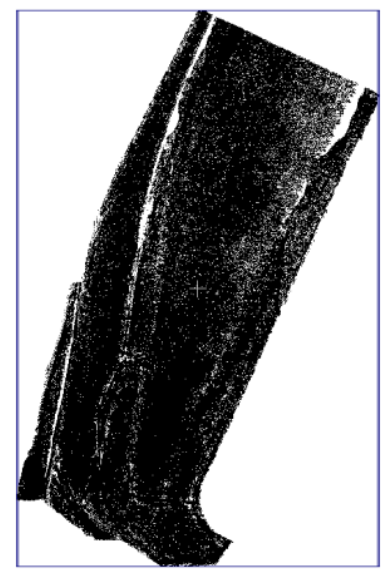

Figure 24. The result for match bounding-box centers registration method - the registered point cloud data

Table 5 below shows the summary of the parameters involve in ICP registration method.

\begin{tabular}{|c|c|}
\hline \multicolumn{2}{|c|}{ Parameters for ICP Registration Method } \\
\hline Parameters & Value \\
\hline Number of iteration & 30 \\
\hline Random sampling unit & 300,000 \\
\hline Rotation & XYZ \\
\hline Translation & XYZ \\
\hline Calculated Parameters for ICP Registration Method \\
\hline Scale & 1.00319 \\
\hline RMS (computed on 231,410 points) & 0.10891 \\
\hline
\end{tabular}

Table 5. Summary of the parameters involve in ICP registration method

While, Figure 25 and Figure 26 shows the computed applied transformation matrix and the registered point clouds data for ICP registration method, respectively.

\begin{tabular}{|c|}
\hline $1.002-0.0020 .018-32.341$ \\
\hline $0.0021 .003-0.003-436.887$ \\
\hline $\begin{array}{l}-0.0180 .0031 .0031015 .764 \\
\end{array}$ \\
\hline 0.0000 .0000 .0001 .00 \\
\hline
\end{tabular}

Figure 25. Computed applied transformation matrix for ICP registration method

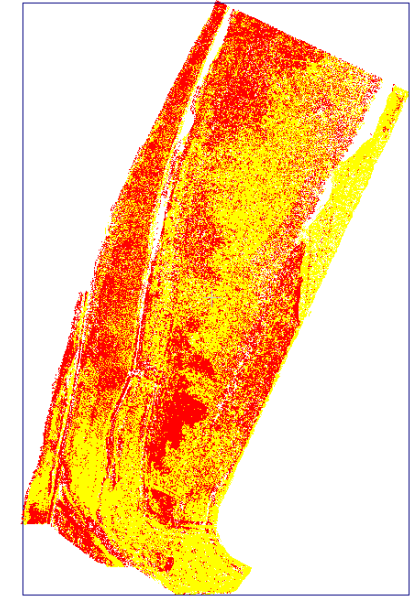

Figure 26. The result for ICP registration method - the registered point cloud data

The results gathered from the $\mathrm{MBBC}$ and ICP registration methods were than used in the 3D surface deviation analysis.

\subsection{D surface deviation analysis results}

For each $\mathrm{C} 2 \mathrm{C}$ distance computation method, CloudCompare software will compute four values for the distance which are the maximum distance, average distance, mean distance and the standard deviation. Table 6 below shows the example of the computed values for nearest neighbor $\mathrm{C} 2 \mathrm{C}$ distance computation for MBBC registration method.

\begin{tabular}{|c|c|c|}
\hline \multicolumn{3}{|c|}{$\begin{array}{c}\text { Computed Values for nearest neighbor C2C Distance for } \\
\text { MBBC Registration Method }\end{array}$} \\
\hline $\begin{array}{c}\text { Compute Distances } \\
(\mathrm{m})\end{array}$ & $\begin{array}{c}\text { Mean Distance: } \\
0.286223\end{array}$ & $\begin{array}{c}\text { Standard Deviation: } \\
0.175165\end{array}$ \\
\hline Max distance (m) & \multicolumn{3}{|c|}{1.63581908} \\
\hline Average distance (m) & \multicolumn{3}{|c|}{0.188983} \\
\hline
\end{tabular}

Table 6. Computed values for nearest neighbor $\mathrm{C} 2 \mathrm{C}$ distance computation for $\mathrm{MBBC}$ registration method

While, Table 7 below shows mean distance and standard deviation values for $\mathrm{C} 2 \mathrm{C}$ distance computations (including the local surface models) for MBBC registration method.

\begin{tabular}{|c|c|c|}
\hline \multicolumn{3}{|c|}{ Local model methods } \\
\hline C2C methods & Mean distance & Standard deviation \\
\hline $\begin{array}{c}\text { Least square } \\
\text { plane }\end{array}$ & 0.233652 & 0.180186 \\
\hline $\begin{array}{c}\text { 2D1/2 } \\
\text { triangulation }\end{array}$ & 0.280690 & 0.178398 \\
\hline Quadric & 0.251922 & 0.183940 \\
\hline Nearest neighbor & 0.286223 & 0.175165 \\
\hline
\end{tabular}

Table 7. Mean distance and standard deviation values for $\mathrm{C} 2 \mathrm{C}$ distance computations for MBBC registration method

The bar graph in Figure 27 below shows the comparison of mean distance and standard deviation values for the $\mathrm{C} 2 \mathrm{C}$ distance computation for MBBC registration method. 


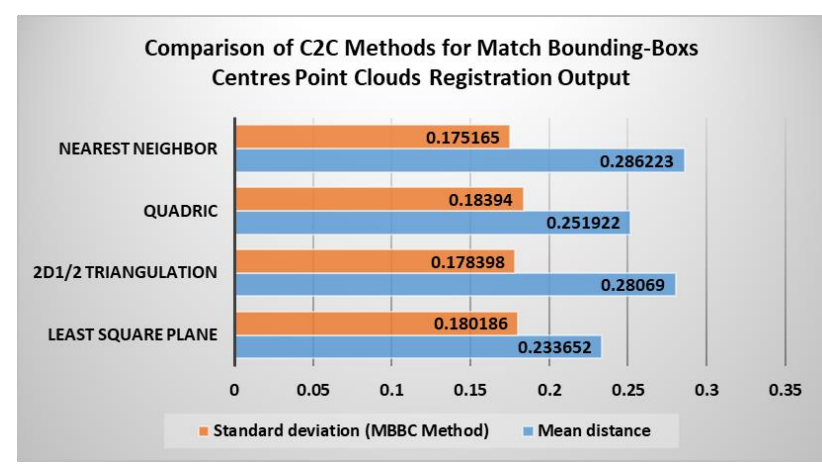

Figure 27. Comparison of mean distance and standard deviation values for the $\mathrm{C} 2 \mathrm{C}$ distance computation for $\mathrm{MBBC}$ registration method

Figure 27 (above) shows the differences in mean distance and standard deviation values for all $\mathrm{C} 2 \mathrm{C}$ distance computation methods. The nearest neighbor method shows the smallest standard deviation value as compared to other methods. This result gives an indicator that the best $\mathrm{C} 2 \mathrm{C}$ distance computation method for MBBC registration output for MLS datasets is the nearest neighbor.

Table 8 below shows the example of the computed nearest neighbor $\mathrm{C} 2 \mathrm{C}$ distance computation for ICP registration method. While, Table 9 below shows mean distance and standard deviation values for $\mathrm{C} 2 \mathrm{C}$ distance computations (including the local surface models) for ICP registration method.

\begin{tabular}{|c|c|c|}
\hline \multicolumn{3}{|c|}{$\begin{array}{c}\text { Computed Values for nearest neighbor C2C Distance for } \\
\text { ICP Registration Method }\end{array}$} \\
\hline $\begin{array}{c}\text { Compute } \\
\text { Distances }(\mathrm{m})\end{array}$ & $\begin{array}{c}\text { Mean Distance: } \\
0.171775\end{array}$ & $\begin{array}{c}\text { Standard Deviation: } \\
0.176260\end{array}$ \\
\hline Max distance $(\mathrm{m})$ & \multicolumn{2}{|c|}{1.645252} \\
\hline $\begin{array}{c}\text { Average distance } \\
(\mathrm{m})\end{array}$ & \multicolumn{2}{|c|}{0.0875221} \\
\hline
\end{tabular}

Table 8. Computed Values for nearest neighbor $\mathrm{C} 2 \mathrm{C}$ distance computation for ICP registration method

\begin{tabular}{|c|c|c|}
\hline \multicolumn{3}{|c|}{ Local model methods } \\
\hline C2C methods & Mean distance & $\begin{array}{l}\text { Standard } \\
\text { deviation }\end{array}$ \\
\hline Least square plane & 0.129874 & 0.165488 \\
\hline 2D1/2 triangulation & 0.159628 & 0.177548 \\
\hline Quadric & 0.143082 & 0.176709 \\
\hline Nearest Neighbor & 0.171775 & 0.176260 \\
\hline
\end{tabular}

Table 9. Mean distance and standard deviation values for $\mathrm{C} 2 \mathrm{C}$ distance computations for ICP registration method

The bar graph in Figure 28 below shows the comparison of mean distance and standard deviation values for the $\mathrm{C} 2 \mathrm{C}$ distance computation for ICP registration method.

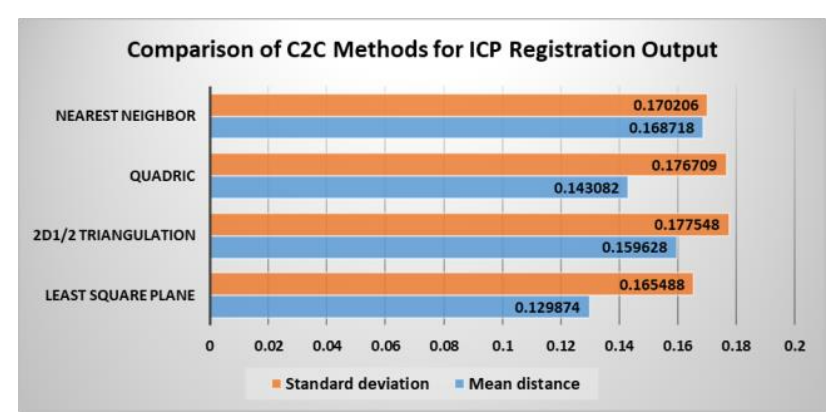

Figure 28. Comparison of mean distance and standard deviation values for the $\mathrm{C} 2 \mathrm{C}$ distance computation for ICP registration method

Figure 28 (above) shows the differences in mean distance and standard deviation values for all $\mathrm{C} 2 \mathrm{C}$ distance computation methods. The least square plane method shows the smallest standard deviation value as compared to other methods. This result gives an indicator that the best $\mathrm{C} 2 \mathrm{C}$ distance computation method for ICP registration output for MLS datasets is the least square plane.

Figure 29 below shows the example of nearest neighbor C2C distance computation results for both MBBC and ICP registration methods for the study area.

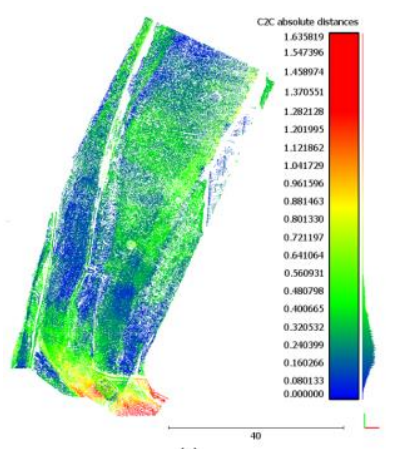

(a)

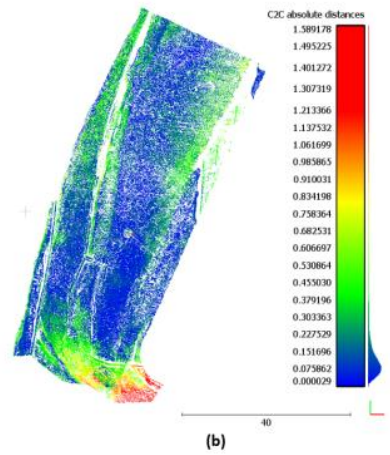

(b)
Figure 29. The nearest neighbor $\mathrm{C} 2 \mathrm{C}$ distance computation results - (a) $\mathrm{C} 2 \mathrm{C}$ result from the $\mathrm{MBBC}$ registration output, (b) $\mathrm{C} 2 \mathrm{C}$ result from the ICP registration output

The above figures show that there is a movement occurs at the study area. The highest movement happened at the area with the red colour. It can be clearly seen that both MBBC and ICP methods shows almost similar results especially at the area with the highest movement value. The questions arise here are (a) which point clouds registration method is the most suitable method for MLS datasets? and (b) which $\mathrm{C} 2 \mathrm{C}$ distance computation method is the best method that can give the best results for detecting movement at the landslide area via MLS datasets?.

\subsection{Overall analysis of the results}

The overall analysis of the results obtained from the research was carried out in two ways, which are (a) the comparison analysis between the $\mathrm{C} 2 \mathrm{C}$ results from the MBBC and ICP methods using mobile laser scanning data with the $\mathrm{C} 2 \mathrm{C}$ result that was generated from the UAV-Photogrammetric data, and (b) the comparison analysis between the four $\mathrm{C} 2 \mathrm{C}$ computation 
methods that was finally give the finding on which registration methods that was suitable to be used in analysing the landslide movement from mobile laser scanning data.

\subsubsection{The comparison analysis using UAV- Photogrammetric Data}

In this research, the $\mathrm{C} 2 \mathrm{C}$ result that was generated from the UAV-Photogrammetric data was selected as benchmark data to evaluate the $\mathrm{C} 2 \mathrm{C}$ results from both $\mathrm{MBBC}$ and ICP methods. Figure 30 shows the comparison analysis between the $\mathrm{C} 2 \mathrm{C}$ distance computation results that was generated from the UAVphotogrammetric data and $\mathrm{C} 2 \mathrm{C}$ distance computation results that was generated from mobile laser scanning data.
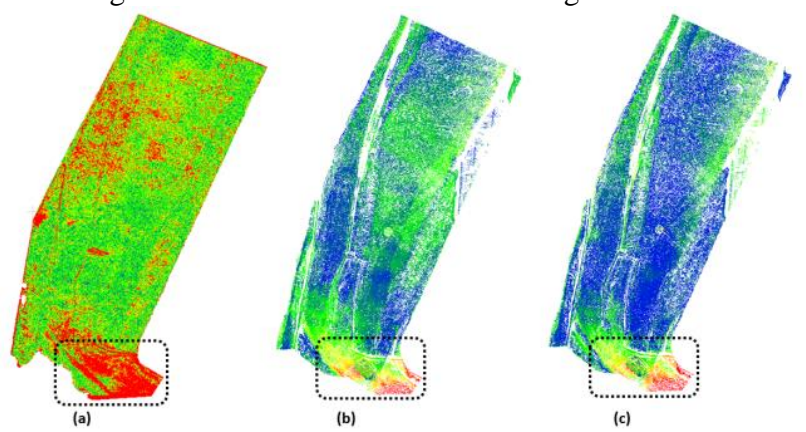

Figure 30. The comparison analysis between the outputs of cloud to cloud distance computations - (a) $\mathrm{C} 2 \mathrm{C}$ results from UAV-Photogrammetric data, (b) $\mathrm{C} 2 \mathrm{C}$ result from mobile laser scanning data for match bounding-box centres method and (c) $\mathrm{C} 2 \mathrm{C}$ result from mobile laser scanning data for ICP method

According to Figure 30, both $\mathrm{C} 2 \mathrm{C}$ results (as generated from the MBBC and ICP registration methods) was found similar with the $\mathrm{C} 2 \mathrm{C}$ result that was generated from UAVPhotogrammetric data. The finding also concludes that both MBBC and ICP methods can be used to detect the movement of the landslide surface.

\subsubsection{The analysis of $\mathrm{C} 2 \mathrm{C}$ distance computation methods}

The second analysis involves with the analysis of all the $\mathrm{C} 2 \mathrm{C}$ distance computation methods (least square plane, 2D1/2 triangulation, quadric and nearest neighbor) which refers to the average standard deviation values that was generated from the MBBC and ICP registration outputs (as shown in Table 10). While Figure 31 shows the statistical plot for the comparison analysis of the $\mathrm{C} 2 \mathrm{C}$ accuracy between MBBC and ICP registration methods.

\begin{tabular}{|c|c|c|}
\hline $\begin{array}{c}\text { C2C computation } \\
\text { methods }\end{array}$ & $\begin{array}{c}\text { Average } \\
\text { standard } \\
\text { deviation values } \\
\text { (MBBC } \\
\text { Method) }\end{array}$ & $\begin{array}{c}\text { Average } \\
\text { standard } \\
\text { deviation values } \\
\text { (ICP Method) }\end{array}$ \\
\hline Least square plane & 0.180186 & 0.165488 \\
\hline 2D1/2 triangulation & 0.178398 & 0.177548 \\
\hline Quadric & 0.183940 & 0.176709 \\
\hline Nearest neighbor & 0.175165 & 0.170206 \\
\hline
\end{tabular}

Table 10. The average $\mathrm{C} 2 \mathrm{C}$ distance computation standard deviation values between MBBC and ICP methods

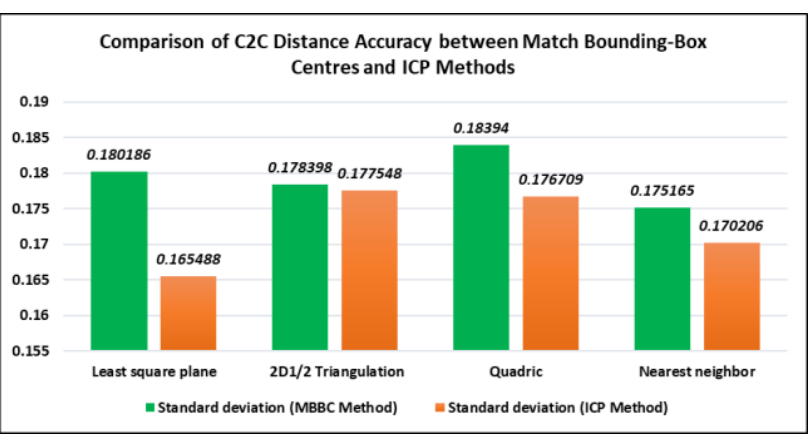

Figure 31. The comparison of $\mathrm{C} 2 \mathrm{C}$ distance accuracy between $\mathrm{MBBC}$ and ICP registration methods

Figure 31 shows that the ICP registration method prove to be the best method to be used to detect and to monitor the movement of the landslide area using mobile laser scanning data. The least square plane $\mathrm{C} 2 \mathrm{C}$ distance computation method shows the lowest standard deviation value and also prove to be the most suitable method to be implemented for the detection of the changes between the two epoch's mobile laser scanning datasets.

\section{CONCLUSION}

As mentioned at the beginning of the paper, the aim of the research is to find the best point clouds registration and cloudto-cloud distance computation methods for the change detection analysis of landslide movement from the data acquired using mobile laser scanning system. It can be concluded that the Iterative Closest Point (ICP) registration method and Least Square Plane $\mathrm{C} 2 \mathrm{C}$ distance method were the best method among others in terms of obtained better accuracy for the 3D landslide surface change detection of Mobile Laser Scanning data between two epochs. In the future, the results from this research can be used as a guideline especially in landslide movement change detection analysis and also for various kind of applications that handled with 3D surface data.

\section{ACKNOWLEDGEMENTS}

This research is part of the Universiti Teknologi Malaysia Research Grant under the vot number 19H69.

\section{REFERENCES}

Barnhart, T. B., and Crosby, B. T., 2013. Comparing two methods of surface change detection on an evolving thermokarst using high-temporal-frequency terrestrial laser scanning, Selawik River, Alaska. Remote Sensing, 5(6), 2813-2837.

Haugen, B. D., 2016. Qualitative and quantitative comparative analyses of 3D lidar landslide displacement field measurements: Colorado School of Mines.

Jafari B.M., 2016. Deflection Measurement Through 3D Point Cloud Analysis. Thesis. Master of Science Civil and Infrastructure Engineering. George Mason University. 2016.

Manousakis J, Zekkos D, Saroglouc H and Clark M., 2016. Comparison of UAV-Enabled Photogrammetry-Based 3D Point 
Clouds and Interpolated DSMs of Sloping Terrain for Rockfall Hazard Analysis. The International Archives of the Photogrammetry, Remote Sensing and Spatial Information Sciences, Volume XLII-2/W2, 2016 11th 3D Geoinfo DConference, 20-21 October 2016, Athens, Greece

Moghaddame-Jafari, B., 2017. Deflection Measurement through 3D Point Cloud Analysis.

Oniga, E., Savu, A., and Negrila, A., 2016. The Evaluation of Cloudcompare Software in The Process of Tls Point Clouds Registration (Vol. 21)

Shen Y, Lindenbergh R and Wang J., 2017. Change Analysis in Structural Laser Scanning Point Clouds: The Baseline Method. Sensors 2017, 17, 26; doi:10.3390/s17010026

Revised August 2018 